Revue

Revue de l'histoire des religions

de Ihistoire

des religions

1| 2011

Varia

Marie-Hélène BLANCHET, Georges-Gennadios Scholarios (vers 1400-vers 1472). Un intellectuel orthodoxe face à la disparition de l'empire byzantin

Paris, Éditions de l'Institut Français d'Études Byzantines (« Archives de

l'Orient chrétien ", 20), 2008, 540 p., $24 \mathrm{~cm}, 80 €$.

Michel Stavrou

\title{
OpenEdition
}

Journals

Édition électronique

URL : http://journals.openedition.org/rhr/7735

DOI : $10.4000 /$ rhr.7735

ISSN : 2105-2573

Éditeur

Armand Colin

Édition imprimée

Date de publication : 1 mars 2011

Pagination : 120-124

ISBN : 978-2200-92685-4

ISSN : 0035-1423

Référence électronique

Michel Stavrou, « Marie-Hélène BLANCHET, Georges-Gennadios Scholarios (vers 1400-vers 1472). Un intellectuel orthodoxe face à la disparition de l'empire byzantin », Revue de l'histoire des religions [En ligne], 1 | 2011, mis en ligne le 30 mai 2011, consulté le 09 octobre 2020. URL : http:// journals.openedition.org/rhr/7735; DOl : https://doi.org/10.4000/rhr.7735

Ce document a été généré automatiquement le 9 octobre 2020.

Tous droits réservés 


\section{Marie-Hélène BLANCHET, Georges- Gennadios Scholarios (vers 1400-vers 1472). Un intellectuel orthodoxe face à la disparition de l'empire byzantin}

Paris, Éditions de l'Institut Français d'Études Byzantines (« Archives de l'Orient chrétien ", 20), 2008, 540 p., $24 \mathrm{~cm}, 80 €$.

\section{Michel Stavrou}

\section{RÉFÉRENCE}

Marie-Hélène BLANCHET, Georges-Gennadios Scholarios (vers 1400-vers 1472). Un intellectuel orthodoxe face à la disparition de l'empire byzantin, Paris, Éditions de l'Institut Français d'Études Byzantines (« Archives de l'Orient chrétien », 20), 2008, 540 p., $24 \mathrm{~cm}, 80 €$.

Gennade II Scholarios est passé aux yeux de la postérité comme le premier patriarche de Constantinople de l'époque de la domination ottomane, celui qui sauva héroïquement le Patriarcat après la chute de l'Empire byzantin en 1453. On savait moins que Gennade II (Georges avant sa profession monastique) avait été auparavant un intellectuel impliqué aux côtés de l'empereur Jean VIII dans le dialogue théologique avec les Latins, notamment lors du concile d'union de Ferrare-Florence (1438-1439), avant de diriger de 1446 à 1453 la Synaxe - faction byzantine anti-unioniste hostile à la politique proromaine de Constantin XI -, ce qui lui vaudra d'être choisi par le sultan Mehmet II comme patriarche et donc, de facto, comme ethnarque des chrétiens (Rum). C'est à ce personnage complexe, voire contradictoire, et aux énigmes nombreuses qui jalonnent son parcours de vie sinueux et jalonné de revirements, qu'est consacré l'ouvrage savant de Marie-Hélène Blanchet, publié au terme d'une thèse de doctorat soutenue à l'Université Toulouse II Le Mirail. Cette étude approfondie met de la lumière et de l'ordre dans un dossier historique particulièrement complexe. 
2 Après un important préambule historiographique, où l'auteur montre que Gennade Scholarios, comme Nicéphore Blemmydès ( xIII $^{\mathrm{e}} \mathrm{s}$.), a fait l'objet - en raison de ses écrits contrastés - d'une vaine querelle d'appropriation entre historiens catholiques et orthodoxes (ainsi le P.Théodore Zissis, de l'université de Thessalonique, nie l'authenticité des écrits pro-unionistes de Scholarios), l'ouvrage est structuré sous forme d'un diptyque composé d'une première partie sur la carrière patriarcale de Scholarios (1454-1456) et d'une seconde partie consacrée à Scholarios et à l'union des Églises (avant 1453). Ce plan, choisi par l'auteur qui adopte ainsi une chronologie inversée pour éclairer selon une « démarche démystificatrice » la période qui précède le patriarcat à la lumière de ce que nous révèlent les événements ultérieurs, est a priori audacieux et déroutant pour le lecteur, mais il révèle finalement toute sa pertinence. La mise en lumière d'hésitations du patriarche Gennade et d'erreurs fatales dans son administration du patriarcat nous permet de mieux comprendre des événements de la période antérieure de sa vie. L'étude d'ensemble, riche et bien documentée, apporte, au terme d'une analyse de l'A., une vision renouvelée du parcours et de l'évolution intérieure du patriarche.

3 Dans la première partie, l'auteur décrit les événements allant de la chute de Constantinople à l'accession de Gennade II au patriarcat, puis le statut du patriarche orthodoxe dans l'Empire ottoman. Elle souligne que Mehmet II a restauré le patriarcat de facto à travers le berat (avec les droits afférents) délivré au nouveau patriarche, mais non de jure parce que le droit musulman ne connaissait pas la notion de personne morale, et que le sultan ne pouvait donc rien accorder au patriarcat en tant que tel (p. 86). Notons que, pour que l'élection de Scholarios fût valable, la présence des métropolites du synode était indispensable, pas seulement pour déclarer vacant le siège patriarcal, comme l'écrit l'auteur (p. 80), mais pour procéder à l'élection du patriarche, même de façon purement formelle. On se trouve alors dans une situation totalement nouvelle puisque l'empire chrétien a disparu et son chef, l'empereur, avec lui. Pour justifier l'idée que Scholarios concevait son patriarcat comme le commencement d'une ère nouvelle, l'auteur invoque (p. 119, n. 40) une "étonnante expression » du Premier Discours de Théodore Agallianos, le plus proche conseiller de Scholarios (identifié à l'évêque Théophane de Médie), qui qualifie Gennade de "premier patriarche et didascale de la terre entière ». Or l'adjectif premier n'a pas ici de valeur temporelle mais désigne classiquement la primauté du patriarche de Constantinople au sein de la communion des quatre patriarches orthodoxes d'Orient. Le patriarche conçoit son ministère comme celui d'un "père" et "médiateur sur terre" pour tous les orthodoxes (p.115), ce qui, quoi qu'en dise l'auteur, ne se démarque guère de la conception ancienne, présentée dans l'Eisagogè de Photius, d'un patriarche «image vivante et animée du Christ ». En revanche, il est singulier que Gennade II signe la plupart de ses textes d'une formule très sobre : "Gennade le patriarche ». Il est clair que pour lui - comme à l'origine -, le qualificatif cecuménique est relatif à l'empire romain chrétien, lui-même virtuellement cecuménique, c'est-à-dire universel (les patriarches suivants reprendront cette titulature en un sens nouveau). Et Gennade, dans une lettre privée rédigée en 1455 , résume de manière prophétique la nouvelle situation historique en des termes encore actuels: «Aujourd'hui le christianisme est semblable à celui d'avant Constantin : en effet, nous n'avons aujourd'hui ni Empire, ni Église libre, ni expression libre... » (p. 119). Le seul horizon des chrétiens orthodoxes est désormais la "patrie éternelle» qui vaut toutes les souffrances (p.126). L'idée de l'auteur qu'après la chute de Byzance, l'Église orthodoxe est, sous les Ottomans, 
désormais libérée des «compromis de l'époque précédente avec le pragmatisme politique " (p.67), procède d'un curieux angélisme qui est d'ailleurs contredit par la mention d'interventions constantes des autorités ottomanes dans le choix des prélats, occasionnant chez les hiérarques d'inévitables compromis, voire des compromissions. Dans cette situation précaire, Scholarios remarque que le "respect minutieux" (leptologia) des règles liturgiques n'est pas absolument impératif puisque les dispositions détaillées n'existaient pas dans l'Église primitive (p. 164), ce qui témoigne non d'un relativisme mais d'un sens historique manifeste chez ce patriarche. Pour lui, l'Église se doit d'adapter sa discipline traditionnelle, par le recours à l'économie, notamment dans le cas des remariages quand le conjoint a disparu dans des événements dramatiques.

4 La conception de l'économie attribuée par l'auteur au droit canon byzantin apparaît un peu caricaturale: "un accommodement ponctuel justifié par une circonstance exceptionnelle» (p.149) ou "une concession temporaire et regrettable»(p.311). L'économie ecclésiale s'inscrit bien dans « la perspective du salut des âmes » mais elle n'implique pas forcément un «assouplissement momentané de la loi ». Ce peut-être même l'inverse (durcissement) si la situation pastorale le requiert car l'Église est placée sous le régime de la grâce et non sous celui de la loi, comme dans l'Ancienne Alliance.

5 L'auteur nous dépeint de manière passionnante les deux années passées par Gennade à la tête du patriarcat, notamment son éradication impitoyable des idées religieuses néoplatoniciennes et néopaïennes du fameux Pléthon (†1452), dont le manuscrit autographe des Lois est brûlé publiquement par Scholarios. Le patriarche poursuit également une politique d'indulgence et de discrétion envers ceux qui s'étaient compromis dans l'unionisme des dernières années de Byzance. Comme le montre bien l'auteur, cela lui sera durement reproché par le clan des zélotes (que l'auteur appelle les «ultra-orthodoxes»), qui désirent des sanctions envers les coupables et une clarification de la position de l'Église orthodoxe sur la question de l'unionisme (sans doute appuyés sur ce point par le pouvoir ottoman qui craint tout rapprochement avec Rome). Finalement, les divisions profondes qui avaient déchiré l'Église byzantine vers 1440 ressurgissent de façon larvée dans ce nouveau contexte. L'attitude fuyante et incohérente de Scholarios, dont les propos antiunionistes étaient pourtant très fermes avant 1453, provoquent, au bout de deux ans, sa démission en 1456, suivie de son exil au monastère de Vatopédi (à l'Athos), puis au monastère Saint-Jean-le-Précurseur près de Serrès, où il mourra vers 1472. L'auteur démontre de façon très convaincante que la thèse de deux retours ultérieurs de Scholarios au patriarcat n'est pas fondée et qu'il vaut mieux considérer que les deux séjours ultérieurs de Scholarios dans la capitale ottomane étaient ceux d'un simple moine érudit.

6 La deuxième partie de l'ouvrage, en revenant en arrière, nous offre une description très intéressante et rigoureuse de l'époque précédant 1453, avec la préparation du concile d'union de Florence, son déroulement et ses répercussions, le tout du point de vue de Scholarios. L'auteur analyse finement le passage progressif de ce conseiller de l'empereur, d'une position de soutien à l'unionisme de Florence, à celle d'opposant, tout en restant attaché à une union possible de tous les chrétiens mais sous d'autres conditions. Son antiunionisme semble avoir été dicté moins par des considérations doctrinales que politiques et surtout culturelles. L'orthodoxie représentait pour Scholarios le ciment unissant le "peuple des Romains » (c'est-à-dire des Byzantins); c'est pourquoi il fallait défendre le «dogme des ancêtres" (patrion dogma). L'auteur 
présente dans le détail et de façon toujours admirablement documentée l'histoire de la Synaxe, cette sorte d'"Église parallèle ", "véritable conservatoire de l'orthodoxie " face à l'unionisme officiel, qui fut dirigée par Marc d'Ephèse avant que celui-ci, à la veille de sa mort en 1445 , ne demande à Scholarios de lui succéder à la tête des antiunionistes.

Il resterait à signaler quelques maladresses ou inexactitudes: Prostatès ne devrait pas être rendu par «chef» (p. 107) mais plutôt par «protecteur» (cf. p. 117). Prodromos (pour saint Jean-Baptiste) devrait être traduit par Précurseur et non par le bien laid Prodrome (p. 16, 34, etc). Le néologisme curieux métoque est forgé (p.172, n. 16) pour désigner le métochion que l'on transpose ainsi habituellement en français. La fête de la théophanie (6 janvier) est confondue (p. 80) avec l'Épiphanie occidentale dont le thème liturgique est tout différent. Les Albanais sont présentés comme des Slaves (p.139). L'auteur évoque «l'incarnation de Jésus» (p. 149) au lieu de celle du Verbe ou Fils de Dieu. L'auteur parle de chrétiens « romains » (p.139) pour désigner les Latins, mais le vocable romains prête à confusion puisque c'est la manière dont se désignent euxmêmes les Byzantins ; d'ailleurs Scholarios (cité en n. 14) parle bien des Latinoi. L'auteur écrit que Scholarios «est tenté d'établir une quasi-équivalence entre aristotélisme et christianisme » (p. 181). On ne voit pas comment ce patriarche aurait placé sur le même plan un système philosophique et la foi chrétienne apostolique. Le $8^{\mathrm{e} C o n c i l e}$ œcuménique - considéré comme tel par la tradition byzantine tardive (Balsamon, Scholarios, etc.) -, n'est pas le concile antiromain de 867 présidé par Photius (p. 240, n. 91) mais celui de 879 qui vit la pleine réconciliation entre Rome et Constantinople.

8 On notera encore quelques points discutables. À propos du sens de l'« œcoumène » qu'a en charge le patriarche Gennade, l'auteur restreint ce terme à l'ensemble des communautés orthodoxes de l'empire ottoman (p.158), alors que la juridiction du patriarche s'étendait aussi à tous les territoires d'Europe orientale non soumis aux Ottomans. Concernant le cérémonial du retour des latinisants à l'église orthodoxe fixé par le concile de 1484, l'auteur cite (p. 241) comme source le canon 95 du $6^{\mathrm{e}}$ Concile œcuménique - en fait il s'agit du concile In Trullo (692) - et en conclut (p. 242) que les anciens unionistes sont assimilés aux Latins et considérés comme hérétiques. Le raisonnement est ici un peu simpliste, et la réalité est plus complexe, concernant les Latins aussi bien que les ex-unionistes qui étaient considérés au plan canonique comme des dissidents. À propos du concile de Florence, l'auteur indique (p. 254 et 353) qu'une messe solennelle fut célébrée à Florence le 6 juillet 1439 conjointement par les Latins et les Grecs pour sanctionner la réconciliation des chrétiens d'Orient et d'Occident. En réalité on sait que les Grecs n'y communièrent pas. On trouve aussi quelques approximations qui résultent de transpositions trop rapides de la théologie latine dans le monde grec: les Byzantins poursuivraient la "validité du dogme» (p. 260), l'ecclésiologie byzantine serait celle du «corps mystique du Christ» (p. 261), toutes catégories propres à la théologie médiévale romaine et étrangères à Byzance. On aurait souhaité, par contre, une recherche plus approfondie que la trop maigre note 46 (p. 361) sur la généalogie du concept de latinophronie à Byzance - qui remonte à l'époque du concile d'union de Lyon II (1274). Désignant, de façon assez péjorative, les Byzantins favorables à l'union religieuse avec l'Église latine, cette catégorie était en effet devenue capitale au $\mathrm{xv}^{\mathrm{e}} \mathrm{s}$. Sur Richard Simon, l'auteur renvoie à l'article du DTC $(14 / 2,1941)$ de A. Molien, largement dépassé. Sur Jean Bekkos (p. 243, n. 103), l'auteur aurait pu mentionner l'ouvrage récent d'Alexandra Riebe: Rom in Gemeinschaft mit 
Konstantinopel : Patriarch Johannes XI Bekkos als Verteidiger der Kirchenunion von Lyon (1274), Wiesbaden, 2005.

9 Ces quelques petits défauts restent exceptionnels. La documentation historique concernant les événements retracés et la vie de Scholarios est particulièrement fournie, et l'auteur en fait usage de manière très exhaustive, en analysant avec soin chaque document, et en vérifiant avec rigueur (voire en réfutant) les avis et arguments exprimés par les historiens précédents.

Outre une bibliographie complète, un index des œuvres de Scholarios et un index des noms propres très appréciable, l'ouvrage comporte en annexes: 1) des cartes de l'Empire byzantin de Constantinople ; 2) une chronologie générale, une chronologie des patriarches de Constantinople (1454-1486) ; 3) un tableau des signataires orthodoxes du concile d'union de Florence (1439), plusieurs tableaux des opposants déclarés à l'Union de Florence (1445-1453), quelques données prosopographiques sur les principaux antiunionistes; et 4) une présentation générale des textes de Scholarios, suivie d'une analyse détaillée de cinq textes autobiographiques du patriarche, rédigés entre 1440 et 1467. Ces diverses annexes offrent une mine d'informations pour guider le lecteur et rendre encore plus attrayante la lecture de cet ouvrage à la fois accessible et de haute qualité scientifique.

\section{AUTEURS}

\section{MICHEL STAVROU}

Institut Saint-Serge, Paris. 